\title{
A chaotic dynamical approach to simulate heterogeneous groundwater flow movement
}

\author{
Zhang Cun ${ }^{\mathrm{a}}$, Zhang Wenxian ${ }^{\mathrm{b}}$, Li Yuqing ${ }^{\mathrm{c}}$ \\ XiZang Agriculture and Animal Husbandry College, Linzhi , XiZang, 806000

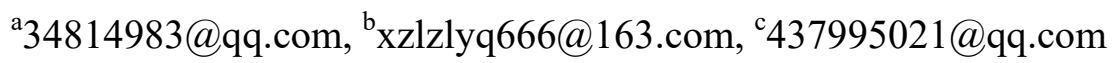

\begin{abstract}
Keywords: chaotic dynamical approach, groundwater flow, numerical simulation, heterogeneous aquifer.

Abstract. The objective of this study was to simulate the groundwater flow movement using a chaotic dynamical approach. The relationship between the actual flow velocity and the mean velocity was described using a random cascade model with branching number of 2 . Results showed that the heterogeneous groundwater flow movement meets the criteria of a nonlinear dynamic system. The use of nonlinear dynamic method improved the accuracy of predicting groundwater flow process using convectional volume averaged Darcy's law.
\end{abstract}

\section{Introduction}

Although the possibility of chaos is expected to occur in partially saturated, heterogeneous structure soil and fractured rock with discontinuity effects and drastic differences in permeability and flow mechanisms, analysis of groundwater flow in heterogeneous aquifer demonstrated the absence of chaos for groundwater flow as related to the impossibility of closed flow paths [1-4]. A common equation used to describe groundwater flow is the cubic law. However, a combination of many nonlinear factors and processes in the heterogeneous media leads to the departure from the cubic law even for flow through a single path. The groundwater flow process can be treated as a dynamic system $[5,6]$. A dynamic system was defined as a physical system with a variation of system parameters. Dynamic system is classified into two types, the deterministic system and the stochastic system [7]. The deterministic systems are driven by a forcing function described explicitly to simulate the evolution of the system. While the stochastic system is driven by the random force described using probabilistic functions [8]. In this study, the heterogeneous groundwater flow was described using a 
chaotic method in which groundwater system was nonlinearly, and coupled effects of several nonlinear processes were governed by nonlinear partial differential equations.

\section{Material and Methods}

The variable hydraulic head field is evaluated numerically by solving the following steady state two-dimension groundwater equation for a heterogeneous confine aquifer.

$$
\frac{\partial}{\partial x}\left(K_{x} \frac{\partial \phi(x, z)}{\partial x}\right)+\frac{\partial}{\partial z}\left(K_{z} \frac{\partial \phi(x, z)}{\partial z}\right)=0
$$

The average velocity of Lagrangean particles is governed by the Darcy equation:

$$
\begin{aligned}
& \bar{v}_{x}=-\frac{K_{x}}{e} \frac{\partial \phi(x, z)}{\partial x} \\
& \bar{v}_{z}=-\frac{K_{z}}{e} \frac{\partial \phi(x, z)}{\partial z}
\end{aligned}
$$

A function $f$ is used to describe possible actual velocity distributions due to soil heterogeneity.

$$
v_{z}=-\frac{1}{e} \frac{K_{z} f \partial \phi}{\partial z}
$$

Integrating Eq. (3) yields:

$$
\frac{1}{\Delta x} \int_{0}^{\Delta x}\left[-\frac{1}{e} \frac{K_{z} f \partial \phi}{\partial z}\right] d x=\bar{v}_{z}
$$

Substituting Eq. (2b) into Eq. (4) yields

$$
\frac{1}{\Delta x} \int_{0}^{\Delta x}\left[-\frac{1}{e} \frac{K_{z} f \partial \phi}{\partial z}\right] d x=-\frac{1}{e} \frac{K_{z} f \partial \phi}{\partial z}
$$

Eq. (5) yields:

$$
\frac{1}{\Delta x} \int_{0}^{\Delta x} f d x=1.0
$$

Fig.1 shows the possible relationship between the actual velocity and the mean velocity in the $z$ direction. Fig. 2 shows the process of construction of a discrete cascade. The mean velocity is successively divided into $b$ equal parts at each step. At the first level, the mean velocity is subdivided into $b=2$ subs, then, at the second level each of the above subs is further subdivided into $b=2$ subs. 


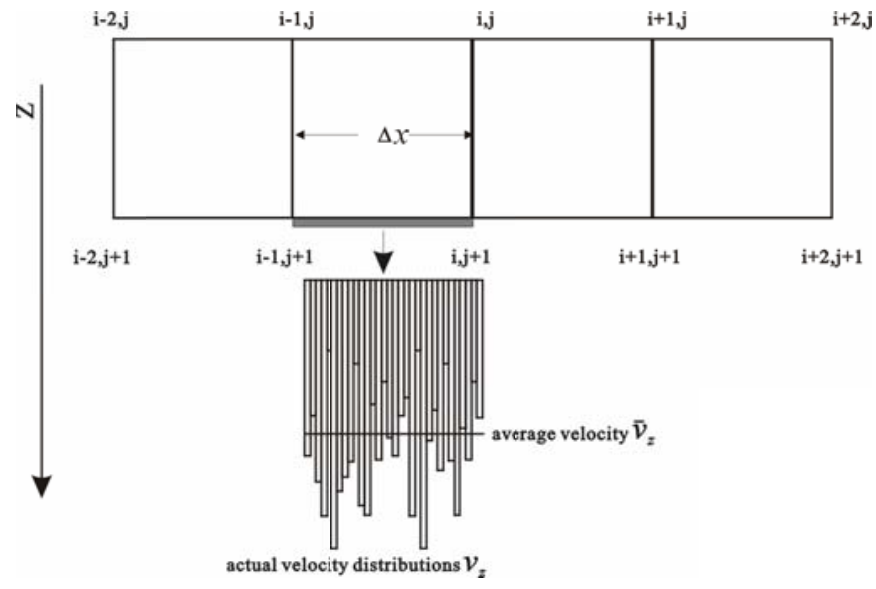

Fig.1 Schematic of possible velocity distributions in the $\mathrm{z}$ direction

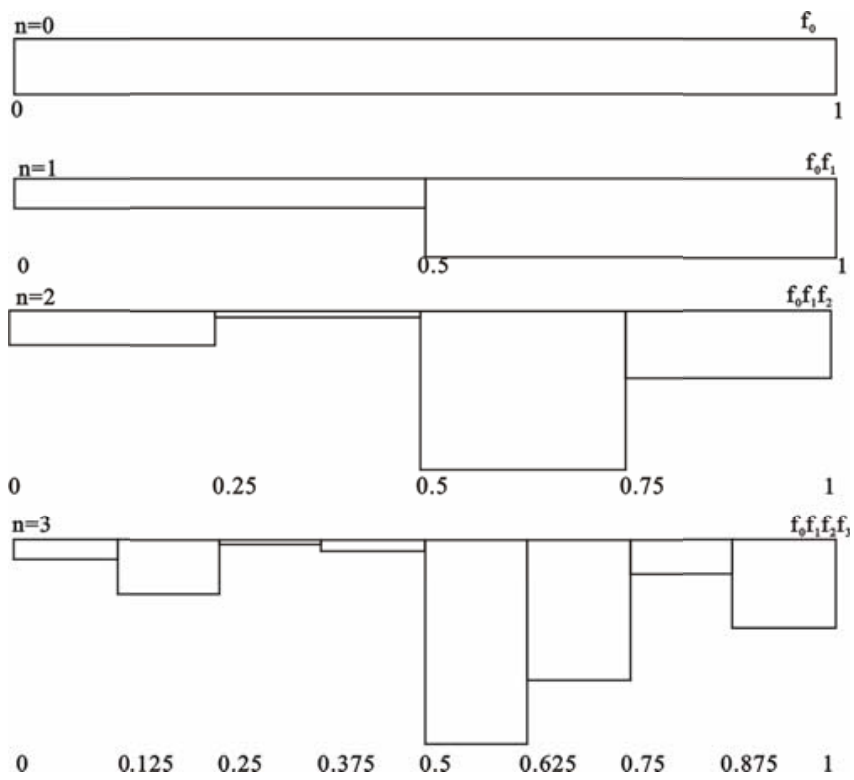

Fig.2 Schematic of discrete random cascade model with branching number $b=2$

Discrete random cascade method [9] is used to generate $f$

$$
f=B Y
$$

Where, B is a generator from the "beta model" and $Y$ is drawn from a lognormal distribution [10]. The beta model is a discrete probability mass function with just two possible outcomes $(B=0$ and $B=b^{\beta}$ ) given by 


$$
\begin{aligned}
& P(B=0)=1-b^{-\beta} \\
& P\left(B=b^{\beta}\right)=b^{-\beta}
\end{aligned}
$$

where $\beta$ is a parameter. $Y$ belongs to the lognormal distribution and can be expressed as:

$$
Y=b^{-\sigma^{2} \ln b / 2+\sigma W}
$$

where $W$ is a standard normal random variate, $\sigma^{2}$ is a parameter equal to the variance of $\log _{b} Y$, with the condition that $E[Y]=1$. The condition $E[f]=1$ is also satisfied. The possibility distribution of Eq.(7) can be expressed as:

$$
\begin{aligned}
& P(f=0)=1-b^{-\beta} \\
& P\left(f=b^{\beta} Y=b^{\beta-\sigma^{2} \ln b / 2+\sigma W}\right)=b^{-\beta}
\end{aligned}
$$

\section{Results and Discussion}

Fig. 3 shows the comparison of simulated potential head in the $x$ direction due to heterogeneous hydraulic conductivity distributions with the numerical method and the random cascade model, respectively. Fig. 4 shows comparison between the calculated velocity of Lagrange particles under the potential head simulated with numerical method and the cascade model, respectively.
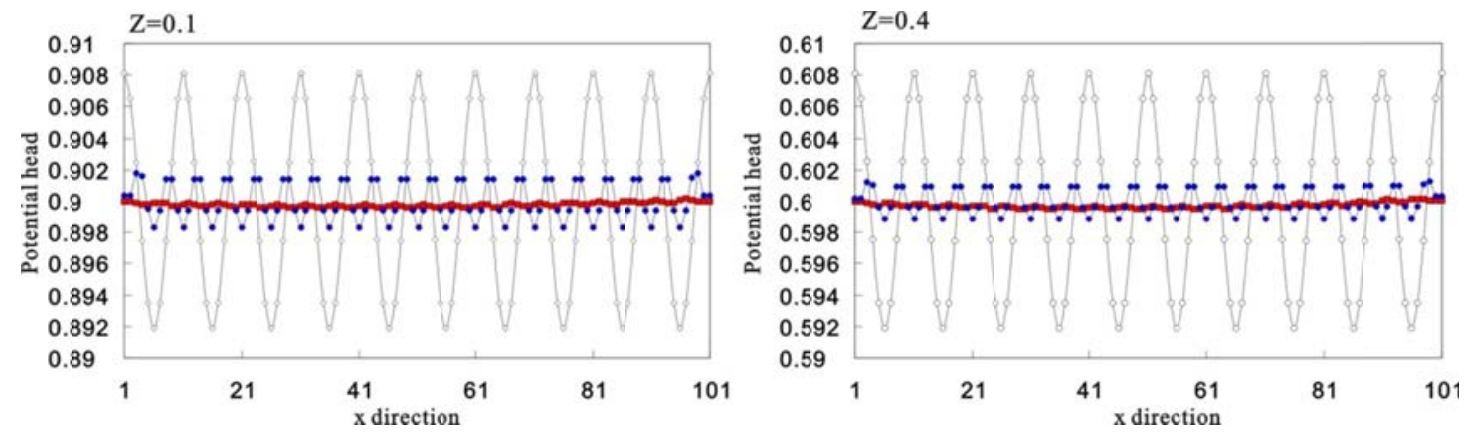

Fig.3 Comparison of potential head simulated with numerical and the random cascade methods at (a) $\mathrm{z}=10$ and (b) $\mathrm{z}=40$, respectively. 

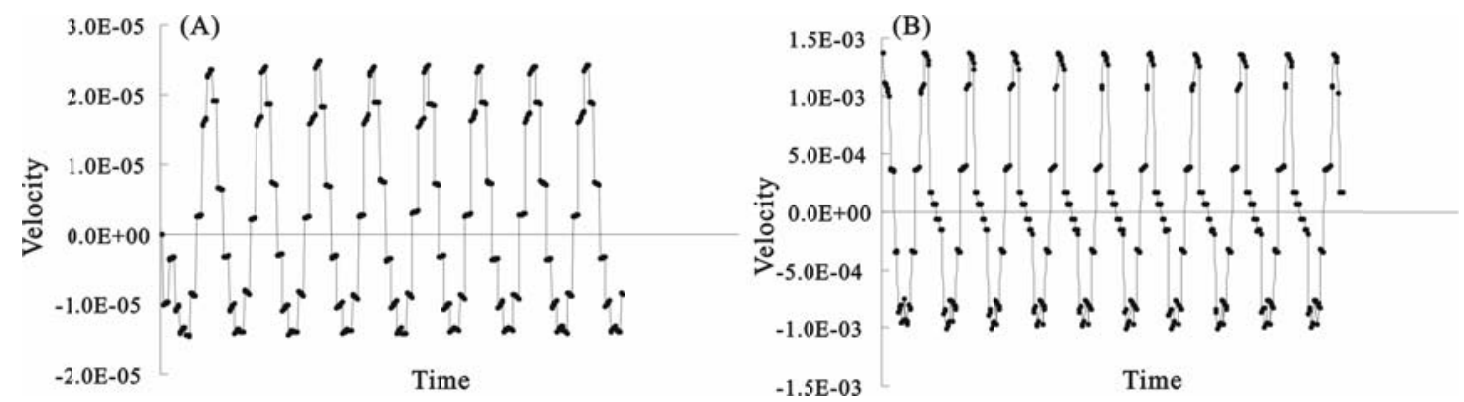

Fig.4 Simulated velocity of Lagrange particle in $\mathrm{x}$ direction under the potential head distributions which was calculated with (a) numerical method (b) the random cascade method.

Comparison between simulated results with the numerical model and the chaotic model (i.e., random cascade model) clearly shows a qualitative similarity in the way the chaotic behaves, indicating that heterogeneous groundwater flow followed routes to and from chaos. As presented in Figs 3 and 4, the flow heterogeneities consisted of two segments, each corresponding to rotation about the unstable fixed point in a given direction. This implies that the attractor has a dimension close but not equal to 2 . These results may also be extended to the case of diffusive tracers, where pore-scale chaotic advection acts to significantly accelerate scalar mixing, essentially enhancing the chaotic mechanism for molecular diffusion.

\section{Conclusions}

The simulations in this study showed that the groundwater flow process meet the criteria of a nonlinear dynamic system. Chaotic fluctuations or water pressure, flow rate were observed. The use of nonlinear dynamic methods improved the accuracy of predicting groundwater flow process using convectional volume averaged Darcy's law.

\section{Acknowledgements}

This work was partly supported by grants from the State's Key Project of Research and Development Plan of China (2016YFC0402405), and Chinese National Natural Science Foundation (Nos. 51379152, 91647109).

Key Special project of Science and Technology Department of Tibet(Z2016C01G01/07);

Key projects of Science and Tech-nology Department of Tibet: Research and its prevention and control technology on agricultural non-point source pollution in Tibe 


\section{References}

[1] Nield, D.A. A. Bejan, 2006. Convection in Porous Media, Springer Verlag, New York.

[2] Vadasz, P. 1999. Local and global transitions to chaos and hysteresis in a porous layer heated from below, Transp. Porous Med. 37:213-245.

[3] Bessa W.M., A.S. de Paula, M.A. Savi, 2009. Chaos control using an adaptive fuzzy sliding mode controller with application to a nonlinear pendulum, Chaos Solitons Fractals 42 :784-791

[4] Kang, P.K., de Anna, P., Nunes, J.P., Bijeljic, B., Blunt, M.J., Juanes, R., 2014. Pore-scale intermittent velocity structure underpinning anomalous transport through 3- d porous media. Geophys. Res. Lett. 41 (17), 6184-6190.

[6] Balasuriya, S., 2015. Dynamical systems techniques for enhancing microfluidic mix- ing. J. Micromech. Microeng. 25 (9). http://dx.doi.org/10.1088/0960-1317/25/9/ 094005 .

[7] Lester, D.R., Metcalfe, G., Trefry, M.G., 2014. Anomalous transport and chaotic advec- tion in homogeneous porous media. Phys. Rev. E 90 (6), 3012 .

[8] Dentz, M., Borgne, T.L., Lester, D.R., de Barros, F.P.J., 2015. Scaling forms of particle densities for Lévy walks and strong anomalous diffusion. Phys. Rev. E 92, 032128 ..

[9] Chatchai, J., Murugesu, S. Neil R.V., 2000. Test of space model of daily rainfall in southwestern Australia bases on non-homogeneous random cascades, Water resource research, 36: 267-284

[10] Schmitt, F. D. Marsan, 2001. Stochastic equations generating continuous multiplicative cascades, Eur.Phys, J.B 20, 3-6 